Karimi, Nader, Mahmoudi , Yasser, and Mazaheri, Kiumars (2014) Temperature fields in a channel partially filled with a porous material under local thermal nonequilibrium condition: an exact solution. Proceedings of the Institution of Mechanical Engineers Part C: Journal of Mechanical Engineering Science . ISSN 0954-4062

Copyright (C) 2014 IMechE

A copy can be downloaded for personal non-commercial research or study, without prior permission or charge

Content must not be changed in any way or reproduced in any format or medium without the formal permission of the copyright holder(s)

When referring to this work, full bibliographic details must be given

http://eprints.gla.ac.uk/91010

Deposited on: 08 April 2014

Enlighten - Research publications by members of the University of Glasgow http://eprints.gla.ac.uk 


\title{
Temperature fields in a channel partially filled with a porous material under local thermal non-equilibrium condition-an exact solution
}

\author{
Y. Mahmoudi, N. Karimi ${ }^{1}$ \\ Department of Engineering, University of Cambridge, Cambridge, United Kingdom
}

\section{Abstract}

The present work examines analytically the forced convection in a channel partially filled with a porous material and subjected to constant wall heat flux. The Darcy-Brikman-Forchheimer model is used to represent the fluid transport through the porous material. The local thermal non-equilibrium, two-equation model is further employed as the solid and fluid energy equations. Two fundamental models (models A and $\mathrm{B}$ ) represent the thermal boundary conditions at the interface between the porous medium and the clear region. The governing equations of the problem are manipulated and for each interface model exact solutions, for the solid and fluid temperature fields, are developed. These solutions incorporate the porous material thickness, Biot number, fluid to solid thermal conductivity ratio and Darcy number as parameters. The results can be readily used to validate numerical simulations. They are, further, applicable to the analysis of enhanced heat transfer, using porous materials, in heat exchangers.

\section{Key words}

porous media, local thermal non-equilibrium, forced convection, heat transfer, exact solution.

\section{Introduction}

The problem of heat transfer enhancement is of rapidly increasing significance [1]. There exist a great number of examples for the heat transfer enhancement by porous materials in natural and manmade

\footnotetext{
${ }^{1}$ Corresponding author, Department of Engineering, University of Cambridge, CB2 1PZ Cambridge, UK, email: nk395@cam.ac.uk
} 
systems [2]. There is also a significant interest in understanding, modelling and simulation of heat and

2 fluid flow in porous media [2]. This is, in part, to improve the predictive tools used in the design of

3 engineering devices in which porous materials are employed to boost the performance. For instance,

4 porous media are widely used in heat exchangers to enhance the heat transfer rate. Although this usually

5 results in a significant increase in the heat transfer rate, it also causes large pressure drops. To avoid such

6 excessive pressure losses sometimes the fluid conduits are filled only partially with porous materials. Due

7 to the existence of a porous-fluid interface in these partially filled systems, their analysis could be more

8 involved compared to the fully filled ones. Further, the recent technological interests in highly efficient

9 energy systems has intensified the need for more accurate heat transfer analyses [3-5]. Accurate

10 prediction of the flow and temperature fields in the partially filled systems heavily depends upon the

11 proper implementation of the boundary conditions on the porous-fluid interface [6]. These include both

12 hydrodynamic and thermal boundary conditions.

13 In general, two different approaches can be undertaken to apply the conservation of energy in flows

14 through porous media. These include the Local Thermal Equilibrium (LTE) and the Local Thermal Non-

15 Equilibrium (LTNE). LTE model assumes the temperature of the fluid and solid phases are locally the same [6-8]. However, LTNE recognises a finite temperature difference between the two phases. When

17 LTE model is in use, the continuity of temperature and heat flux can be utilised as the boundary conditions at the porous-fluid interface. LTNE model, however, requires implementation of an additional thermal boundary condition at the porous-fluid interface $[3,5-7]$. A precise description of the thermal boundary conditions at the porous-fluid interface is yet to be given and the research in this area is ongoing

$21[5,8]$. In the meantime, a number of models have been reported in the literature, which account for the 22 dominant behaviour of the boundary conditions [7]. While these models have been validated against 23 experiments, their applicability is strongly problem dependent and varies with changes in the parameters

24 [7]. The numerical simulations of the partial-porous systems should be, therefore, capable of handling 25 these subtleties. This calls for the comprehensive validation of these tools which, in turn, generates a need 26 for the development of exact solutions of the flow and temperature fields. 
Analysis of convective heat transfer, in general, includes solution of nonlinear partial differential

2 equations governing the transport of momentum and energy [2]. Hence, it is predominantly feasible

3 through numerical methods. Heat transfer in porous media, however, features certain characteristics that,

4 under some conditions, turn the analysis amenable to the analytical approaches. Utilising these

5 characteristics, this paper reports an analytical analysis of the solid and fluid temperature fields in a

6 system partially filled with a porous insert. This takes into account two different, existing models of the

7 thermal boundary conditions at the porous-fluid interface [7]. Local thermal non-equilibrium model is

8 applied through considering two energy equations for both the fluid phase and the solid matrix. This

9 results in the determination of the temperature fields within the solid and fluid phases. One of the primary

10 aims of the current analysis is to produce a means of validation for the numerical simulations of heat

11 transfer in partially filled systems. The exact solutions, developed in this work, are also of physical

12 significance and can be further employed to evaluate the validity of LTE assumption under varying

13 operating and design parameters.

14 Figure 1 shows the schematic of the problem under investigation. Fluid flows into a channel in which a

15 porous material has been inserted at the core. Constant heat flux boundary condition is applied on the

16 channel walls. Due to the symmetry of this configuration only half of the domain is considered in the

17 analysis. The flow is laminar, steady, thermally and hydrodynamically fully developed, and

18 incompressible. Natural convection, viscous heat generation and radiation heat transfer are ignored.

19 Further, the thermo-physical properties of the fluid and solid are assumed constant.

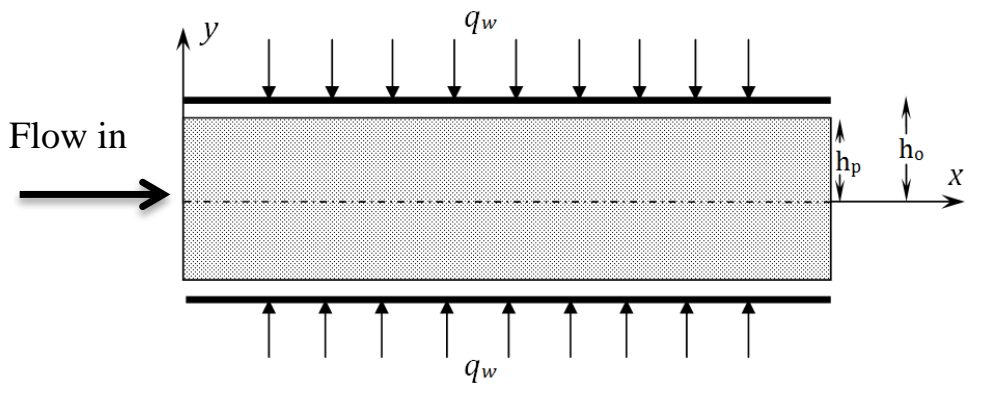


Fig. 1. Schematic of the problem.

\section{2. Results and discussions}

\section{2.1. Governing equations and the boundary conditions}

$4 \quad$ Momentum equation and energy equation in the clear region are respectively expressed by

$$
-\frac{\partial p}{\partial x}+\mu \frac{\partial^{2} u_{f}}{\partial y^{2}}=0, \quad \rho c_{p} u_{f} \frac{\partial T_{f 1}}{\partial x}=k_{f} \frac{\partial^{2} T_{f 1}}{\partial y^{2}} .
$$

5 Previous investigations [4] have shown that within the porous medium and for $D a<10^{-3}$, the inertia term

6 of the momentum equation within the porous medium is negligible. Thus, fluid flow through the porous

7 medium is represented by the Darcian flow model. Momentum equation in the porous region reduces to

$$
-\frac{\partial p}{\partial x}+\mu_{e f f} \frac{\partial^{2} u_{p}}{\partial y^{2}}-\frac{\mu}{K} u_{p}=0
$$

8 Fluid and solid phases energy equations in the porous region are represented by the following coupled 9 partial differential equations

$$
0=k_{s, e f f} \frac{\partial^{2} T_{s}}{\partial y^{2}}-a_{s f} h_{s f}\left(T_{s}-T_{f 2}\right), \quad \quad \rho c_{p} u_{p} \frac{\partial T_{f 2}}{\partial x}=k_{f, e f f} \frac{\partial^{2} T_{f 2}}{\partial y^{2}}+a_{s f} h_{s f}\left(T_{s}-T_{f 2}\right)
$$

The following boundary conditions are applied to the momentum equations,

$$
u_{f}=0 \quad \text { at } \quad y=\mathrm{h}_{0}, \quad u_{f}=u_{p}, \mu_{f} \frac{\partial u_{f}}{\partial y}=\mu_{e f f} \frac{\partial u_{p}}{\partial y} \quad \text { at } \quad y=\mathrm{h}_{\mathrm{p}}, \quad \frac{\partial u_{p}}{\partial y}=0 \quad \text { at } \quad y=0 . \quad(4 \mathrm{a}, \mathrm{b}, \mathrm{c})
$$

11 The boundary conditions applied to the energy equations are

$$
\frac{\partial T_{f 2}}{\partial y}=\frac{\partial T_{s}}{\partial y}=0, \quad \text { at } \quad y=0, \quad k_{f} \frac{\partial T_{f 1}}{\partial y}=q_{w}, \quad \text { at } \quad y=\mathrm{h}_{0}, \quad T_{f 1}=T_{f 2}, \quad \text { at } \quad y=\mathrm{h}_{\mathrm{p}} . \quad(5 \mathrm{a}, \mathrm{b}, \mathrm{c})
$$

12 In the present work, two models (referred to as A and B in the literature) are utilised to describe the 13 temperature at the interface between the clear and porous regions. Model A was proposed by Vafai and co-workers $[8,9]$ and is based upon the division of heat flux between the solid and fluid phases and states

$$
q_{\text {interface }}=\left.k_{f, \text { eff }} \frac{\partial T_{f}}{\partial y}\right|_{\text {interfaee }}+\left.k_{s, \text { eff }} \frac{\partial T_{s}}{\partial y}\right|_{\text {interface }},\left.\quad \quad T_{f}\right|_{\text {ineffice }}=\left.T_{s}\right|_{\text {interface }}=T_{\text {interface }} \text {. }
$$


1 In model $\mathrm{B}$, however, each of the individual phases at the interface receives an equal amount of the heat

2 flux $[8,9]$, thus

$$
q_{\text {interface }}=\left.k_{f, e f f} \frac{\partial T_{f}}{\partial y}\right|_{\text {interface }}=\left.k_{s, e f f} \frac{\partial T_{s}}{\partial y}\right|_{\text {interface }} .
$$

3 In the above equations $q_{\text {interface }}=k_{f}\left(\partial T_{f 1} / \partial y\right)_{y=\mathrm{h}_{\mathrm{p}}}$ and $T_{\text {interface }}$ represents the heat flux and the temperature

4 at the porous-fluid interface. $\mu_{e f f}$ is the effective viscosity of the porous medium which is considered equal

5 to $\mu_{f}$ [10]. The average velocity in the channel is $\bar{u}=1 / \mathrm{h}_{0}\left\{\int_{0}^{\mathrm{h}_{\mathrm{p}}} u_{p} d y+\int_{\mathrm{h}_{\mathrm{p}}}^{\mathrm{h}_{0}} u_{f} d y\right\}$. Integrating Eq. (1b) from $\mathrm{h}_{\mathrm{p}}$

6 to $\mathrm{h}_{0}$ and noting that in the fully developed region $\partial T_{f 1} / \partial x=\partial T_{f 2} / \partial x=\partial T_{f} / \partial x$ are constants, result in

$$
\rho c_{p} \frac{\partial T_{f}}{\partial x} \int_{\mathrm{h}_{\mathrm{p}}}^{\mathrm{h}_{0}} u_{f} d y=\left(q_{w}-q_{\mathrm{interface}}\right)
$$

7 Adding Eqs. (3a) to (3b), integrating the sum from 0 to $h_{p}$ and applying the boundary conditions given by

8 Eqs. (6a) and (6b) reveal the following equation for model A

$$
\rho c_{p} \frac{\partial T_{f}}{\partial x} \int_{0}^{\mathrm{h}_{\mathrm{p}}} u_{p} d y=q_{\mathrm{interface}}
$$

$9 \quad$ Adding Eq. (8) to Eq. (9) and using the definition of the average velocity yield

$$
\left.\rho c_{p} \frac{\partial T_{f}}{\partial x}\right|_{\text {ModelA }}=\frac{q_{\mathrm{w}}}{\mathrm{h}_{0}} \frac{1}{\bar{u}}
$$

10 Substituting Eq. (10) into Eq. (9) renders model A prediction of heat flux at the porous medium-fluid

11 interface as,

$$
\left.\frac{q_{\text {interface }}}{q_{w}}\right|_{\text {ModelA }}=\frac{1}{\mathrm{~h}_{0} \bar{u}} \int_{0}^{\mathrm{h}_{\mathrm{p}}} u_{p} d y .
$$

12 Through adding Eqs. (3a) to (3b), integrating the sum from 0 to $h_{p}$ and applying boundary condition (7)

13 (model B) the following equation is obtained,

$$
\rho c_{p} \frac{\partial T_{f}}{\partial x} \int_{0}^{\mathrm{h}_{\mathrm{p}}} u_{p} d y=2 q_{\text {interface }}
$$

14 Adding Eq. (8) to Eq. (12) and using the definition of average velocity result in 


$$
\left.\rho c_{p} \frac{\partial T_{f}}{\partial x}\right|_{\text {ModelB }}=\frac{1}{\mathrm{~h}_{0} \bar{u}}\left(q_{w}+q_{\text {interface }}\right)
$$

1 An explanation can be given for Eq. (12). The heat flux $q_{\text {interface }}$ from the outer surface is transferred to the

2 porous medium in two different ways. First, conduction in the fluid phase, $q_{f}$, and second, conduction in

3 the solid phase, $q_{s}$. Model B states that each phase receives the same amount of heat flux, which is equal

4 to $q_{\text {interface. }}$ Thus $q_{f}=q_{\text {interface }}$ and $q_{s}=q_{\text {interface }}$. This is in keeping with the second approach of Amiri et. al

5 [9]. The heat transferred to the solid phase is ultimately delivered to the fluid through an internal heat

6 exchange. This heat exchange is, therefore, equal to the heat transferred from the wall to the solid phase

7 via solid conduction, $q_{\text {exchange }}=q_{s}[11]$. It follows that the overall heat transfer to the fluid phase in the

8 porous region is by direct fluid conduction plus the solid conduction and the subsequent internal heat

9 exchange between the solid and fluid. Thus, $q_{\text {total,fluid,porous }}=q_{\text {interface }}+q_{\text {interface }}=2 q_{\text {interface }}$.

10 Substituting Eq. (13) into Eq. (12) reveals model B prediction of the heat flux at the porous medium-

11 fluid interface,

$$
\left.\frac{q_{\text {interface }}}{q_{w}}\right|_{\text {ModelB }}=\frac{\int_{0}^{\mathrm{h}_{\mathrm{p}}} u_{p} \mathrm{~d} y}{2 \mathrm{~h}_{0} \bar{u}-\int_{0}^{\mathrm{h}_{\mathrm{p}}} u_{p} \mathrm{~d} y} .
$$

12 To normalise the governing equations and boundary conditions the following dimensionless variables are 13 introduced.

$$
\begin{gathered}
\left.\Theta\right|_{\text {ModelA }}=\frac{k_{s, e f f}\left(T-T_{\text {interface }}\right)}{q_{\mathrm{w}} \mathrm{h}_{0}},\left.\Theta\right|_{\text {ModelB }}=\frac{k_{s, e f f}\left(T-T_{\mathrm{s}, \text { interface }}\right)}{q_{\mathrm{w}} \mathrm{h}_{0}}, \gamma=\frac{q_{\text {interface }}}{q_{\mathrm{w}}}, k=\frac{k_{s, e f f}}{k_{f, e f f}}, \\
B i=\frac{a_{s f} h_{s f} \mathrm{~h}_{0}^{2}}{k_{s, e f f}}, Y=\frac{y}{\mathrm{~h}_{0}}, S=\frac{\mathrm{h}_{\mathrm{p}}}{\mathrm{h}_{0}}, U=\frac{u}{u_{r}},
\end{gathered}
$$

14 where $u_{r}$ is a characteristic velocity defined as $u_{r}=-\left(\mathrm{h}_{0}^{2} / \mu\right)(\partial p / \partial x)$. The solutions for the momentum 15 Eqs. (1a) and (1b) and the corresponding boundary conditions (4a), (4b), and (4c) are as follows. For the 16 clear region

$$
U_{f}(Y)=-\frac{1}{2} Y^{2}+A Y+B
$$




$$
A=S+\frac{Z \sinh (Z S)\left(S-0.5 \times\left(1+S^{2}\right)+D a\right)}{Z(S-1) \sinh (Z S)-\cosh (Z S)}, \quad B=1 / 2-S-\frac{Z \sinh (Z S)\left(S-0.5 \times\left(1+S^{2}\right)+D a\right)}{Z(S-1) \sinh (Z S)-\cosh (Z S)}
$$

1 Within the porous region

$$
U_{p}=C \cosh (Z Y)+D a, \quad C=\frac{1}{Z \sinh (Z S)} \times(A-S) .
$$

2 By using Eqs. (16a, b, c) and (17a, b) and dimensionless parameters in relation (15a-h) the dimensionless 3 average velocity becomes

$$
\bar{U}=S D a+\frac{D}{Z} \sinh (Z S)-\frac{1}{6}\left(1-S^{3}\right)+\frac{1}{2} A\left(1-S^{2}\right)+B(1-S)
$$

4 where $Z=\sqrt{1 / D a}, S$ is the ratio of the porous medium thickness to the channel height.

5 Equation (11) is converted to non-dimensional form and is combined with Eqs. (17a, b) and (18) 6 revealing

$$
\left.\gamma\right|_{\text {ModelA }}=\frac{1}{\bar{U}} \int_{0}^{s} U_{p} d Y,\left.\quad \gamma\right|_{\text {ModelA }}=\frac{\frac{D}{Z} \sinh (Z S)+S D a}{S D a+\frac{D}{Z} \sinh (Z S)-\frac{1}{6}\left(1-S^{3}\right)+\frac{1}{2} A\left(1-S^{2}\right)+B(1-S)} .
$$

7 Similar to that explained in the derivation of Eq. (19b), an expression for the heat flux at the porous 8 medium-fluid interface for model $\mathrm{B}$, is obtained as

$$
\left.\gamma\right|_{\text {ModelB }}=\frac{\int_{0}^{s} U_{p} d Y}{2 \bar{U}-\int_{0}^{s} U_{p} d Y}
$$

9 Once again, by using Eqs. (16a, b, c) and $(17 \mathrm{a}, \mathrm{b})$ it can be shown that

$$
\left.\gamma\right|_{\text {ModelB }}=\frac{\frac{D}{Z} \sinh (Z S)+S D a}{2\left(S D a+\frac{D}{Z} \sinh (Z S)-\frac{1}{6}\left(1-S^{3}\right)+\frac{1}{2} A\left(1-S^{2}\right)+B(1-S)\right)-\frac{D}{Z} \sinh (Z S)+S D a} .
$$

\section{2.2. Temperature profile for model $A$}

12 The energy equations and the associated boundary conditions are derived through substitution of the 13 dimensionless variables presented in Eqs. (15a-d) into Eqs. (2), (3a) and (3b). Dimensionless energy 14 equation for the fluid in the clear and porous regions become 


$$
\varepsilon k \frac{U_{f}}{\bar{U}}=\Theta_{f 1}^{\prime \prime}(Y), \quad \frac{U_{p}}{\bar{U}}=\frac{1}{k} \Theta_{f 2}^{\prime \prime}(Y)+B i\left(\Theta_{s}(Y)-\Theta_{f 2}(Y)\right) .
$$

1 The solid phase energy equation in the porous region can be written as

$$
0=\Theta_{s}^{\prime \prime}(Y)-B i\left(\Theta_{s}(Y)-\Theta_{f 2}(Y)\right) \text {. }
$$

2 The boundary conditions of the energy equations are

$$
\Theta_{f 1}^{\prime}(1)=\varepsilon k, \quad \Theta_{f 1}(S)=\Theta_{f 2}(S)=\Theta_{s}(S)=0, \quad \Theta_{f 2}^{\prime}(0)=\Theta_{s}^{\prime}(0)=0 .
$$

3 Applying Eqs. (22b) and (23) at $Y=S$ yields

$$
\frac{U_{p}(S)}{\bar{U}}=\frac{1}{k} \Theta_{f 2}^{\prime \prime}(S)+B i\left(\Theta_{s}(S)-\Theta_{f 2}(S)\right), \quad 0=\Theta_{s}^{\prime \prime}(S)-B i\left(\Theta_{s}(S)-\Theta_{f 2}(S)\right) .
$$

4 Using boundary condition (24b) (i.e. $\left.\Theta_{f 2}(S)=\Theta_{s}(S)=0\right)$, Eqs. (25a) and (25b) are converted to

$$
\Theta_{f 2}^{\prime \prime}(S)=k \frac{U_{p}(S)}{\bar{U}}, \quad \Theta_{s}^{\prime \prime}(S)=0 .
$$

5 Differentiation of Eqs. (22b) and (23) with respect to $Y$ and evaluating the results at $Y=0$ yield

6

$$
\Theta_{f 2}^{\prime \prime \prime}(0)=k \frac{U_{p}^{\prime}(0)}{\bar{U}}=\frac{0}{\bar{U}}=0, \quad \Theta_{s}^{\prime \prime}(0)=0 .
$$

$7 \quad$ Differentiating Eqs. (22b) and (23) with respect to $Y$ for two times gives

$$
\frac{U_{p}^{\prime \prime}(Y)}{\bar{U}}=\frac{1}{k} \Theta_{f 2}^{\prime \prime \prime}(Y)+B i\left(\Theta_{s}^{\prime \prime}(Y)-\Theta_{f 2}^{\prime \prime}(Y)\right), \quad \quad 0=\Theta_{s}^{\prime \prime \prime}(Y)-B i\left(\Theta_{s}^{\prime \prime}(Y)-\Theta_{f 2}^{\prime \prime}(Y)\right) .
$$

8 Adding Eq. (22b) to Eq. (23) results in the following second order differential equation

$$
\frac{U_{p}}{\bar{U}}=\frac{1}{k} \Theta_{f 2}^{\prime \prime}(Y)+\Theta_{s}^{\prime \prime}(Y)
$$

9 By writing $\Theta_{s}^{\prime \prime}(Y)$ as $\Theta_{f 2}^{\prime \prime}(Y)$ and substituting the result into Eq. (28a) we arrive at

$$
\Theta_{f 2}^{\prime \prime \prime \prime}(Y)-B i(1+k) \Theta_{f 2}^{\prime \prime}(Y)=\frac{k}{\bar{U}}\left(-B i U_{p}(Y)+U_{p}^{\prime \prime}(Y)\right)
$$

and similarly writing $\Theta_{f 2}^{\prime \prime}(Y)$ as $\Theta_{s}^{\prime \prime}(Y)$ and substituting the result into Eq. (28b) lead to

$$
\Theta_{s}^{\prime \prime \prime}(Y)-B i(1+k) \Theta_{s}^{\prime \prime}(Y)=-\frac{k}{\bar{U}} B i U_{p}(Y)
$$


1 Equations (30) and (31) are two decoupled ordinary differential energy equations representing the

2 transport of energy in the porous region. Integrating the ordinary differential Eq. (22a) results in the

3 following expression for the temperature distribution of the flow in the clear region under model A

$$
\begin{aligned}
&\left.\Theta_{f 1}(Y)\right|_{\text {ModelA }}= \frac{\varepsilon k}{\bar{U}} \\
&\left(-\frac{Y^{4}}{24}+A \frac{Y^{3}}{6}+B \frac{Y^{2}}{2}+\left(\frac{1}{6}-\frac{A}{2}-B+\bar{U}\right) Y+\frac{S^{4}}{24}-A \frac{S^{3}}{6}\right. \\
&\left.-B \frac{S^{2}}{2}-S\left[\frac{1}{6}-\frac{A}{2}-B+\bar{U}\right]\right),
\end{aligned}
$$

$4 \quad$ where $A$ and $B$ are given by Eqs. (16b) and (16c). The temperature distribution in the porous region is 5 found by solving Eqs. (30) and (31) and applying the boundary conditions given by Eqs. (24) and (27).

6 This reveals

$$
\begin{aligned}
\left.\Theta_{f 2}(Y)\right|_{\text {ModelA }} & =\frac{k}{\bar{U}}\left\{\frac{C\left(Z^{2}-B i\right) \times\left[\cosh (Z Y)-\cosh (Z S) \times\left(1+\xi \times Z^{2}\right)\right]}{Z^{2}\left(-\Gamma^{2}+Z^{2}\right)}\right. \\
+ & \left.\frac{D a B i}{\Gamma^{2}} \times\left(-\xi+\frac{Y^{2}}{2}-\frac{S^{2}}{2}\right)+U_{p}(S) \xi\right\}, \\
\left.\Theta_{s}(Y)\right|_{\text {ModelA }} & =-B i \frac{k}{\bar{U}}\left\{\frac{C\left[\cosh (Z Y)-\cosh (Z S) \times\left(1+\xi \times Z^{2}\right)\right]}{Z^{2}\left(-\Gamma^{2}+Z^{2}\right)}\right. \\
- & \left.\frac{D a}{\Gamma^{2}}\left(-\xi+\frac{Y^{2}}{2}-\frac{S^{2}}{2}\right)\right\},
\end{aligned}
$$

$7 \quad$ where $\Gamma=\sqrt{B i(1+k)}$ and $\xi=\left(\frac{\cosh (\Gamma Y)}{\cosh (\Gamma S)}-1\right) / \Gamma^{2}$

8

\section{$9 \quad$ 2.3. Temperature profile for model B}

10 Substation of Eq. (15a) into Eqs. (2), (3a) and (3b) reveals the different forms of the energy equation for

11 the two phases based on model B. Energy equation for the fluid in the clear region then becomes

$$
\varepsilon k\left(1+\frac{1}{\gamma}\right) \frac{U_{f}}{\bar{U}}=\Theta_{f 1}^{\prime \prime}(Y) .
$$

12 Further, fluid and solid phase energy equations in the porous region take respectively the forms of

$$
\frac{U_{p}}{\bar{U}}=\frac{1}{k} \Theta_{f 2}^{\prime \prime}(Y)+B i\left(\Theta_{s}(Y)-\Theta_{f 2}(Y)\right), 0=\Theta_{s}^{\prime \prime}(Y)-B i\left(\Theta_{s}(Y)-\Theta_{f 2}(Y)\right)
$$

13 The associated boundary conditions are 


$$
\begin{gathered}
\Theta_{f 1}^{\prime}(1)=\varepsilon k, \quad \Theta_{f 1}(S)=\Theta_{f 2}(S), \quad \Theta_{f 2}^{\prime}(0)=\Theta_{s}^{\prime}(0)=0, \\
\Theta_{f 2}^{\prime}(s)=\frac{k}{\gamma}, \Theta_{s}^{\prime}(s)=\frac{1}{\gamma}, \Theta_{s}^{\prime \prime}(s)+B i \Theta_{f 2}(s)=0, \Theta_{s}(s)=0 .
\end{gathered}
$$

1 Following the same procedure explained for model A, and taking the second derivative of Eqs. (36a) and 2 (36b) with respect to $Y$, yield

$$
\begin{aligned}
& \Theta_{f 2}^{\prime \prime \prime \prime}(Y)-B i(1+k) \Theta_{f 2}^{\prime \prime}(Y)=\frac{k}{\bar{U}}\left(1+\frac{1}{\gamma}\right)\left(-B i U_{p}(Y)+U_{p}^{\prime \prime}(Y)\right), \\
& \Theta_{s}^{\prime \prime \prime}(Y)-B i(1+k) \Theta_{s}^{\prime \prime}(Y)=-\frac{k}{\bar{U}}\left(1+\frac{1}{\gamma}\right) B i U_{p}(Y) .
\end{aligned}
$$

3 Evaluating the second and third derivatives of $\Theta_{s}$ and $\Theta_{f 2}$ at the symmetry plane $(Y=0)$ by applying Eq.

$4 \quad(37 \mathrm{c})$ reveals

$$
\Theta_{f 2}^{\prime \prime \prime}(0)=k \frac{U_{p}^{\prime}(0)}{\bar{U}}=0, \quad \Theta_{s}^{\prime \prime \prime}(0)=0 .
$$

5 Solving the ordinary differential Eq. (35) results in the following expressions for the temperature 6 distribution for the flow in the clear region

$$
\begin{gathered}
\left.\Theta_{f 1}(Y)\right|_{\text {Model B }}=\varepsilon \psi\left(-\frac{1}{24} Y^{4}+A \frac{Y^{3}}{6}+B \frac{Y^{2}}{2}+O_{1} Y+O_{2}\right), \\
O_{1}=\frac{k}{\psi}-\left(-\frac{1}{6}+\frac{A}{2}+B\right), O_{2}=\frac{\Theta_{f 2}(s)}{\varepsilon \psi}-\left(-\frac{1}{24} S^{4}+A \frac{S^{3}}{6}+B \frac{S^{2}}{2}+O_{1} S\right),
\end{gathered}
$$

7 where $\psi=(k / \bar{U})(1+1 / \gamma)$ and, $A$ and $B$ are respectively given by Eqs. (16b) and (16c). The temperature

8 distribution in the porous region is found by solving Eqs. (38a) and (38b) and applying Eqs. (37) and (39).

9 These render

$$
\left.\Theta_{f 2}(Y)\right|_{\text {ModelB }}=\frac{\phi 1}{\Gamma^{2}} \cosh (\Gamma Y)+\frac{B i D a \psi}{\Gamma^{2}} \frac{Y^{2}}{2}-\frac{C \psi\left(Z^{2}-B i\right)}{Z^{2}\left(Z^{2}-\Gamma^{2}\right)} \cosh (Z Y)+\phi 2,
$$




$$
\begin{gathered}
\left.\Theta_{s}(Y)\right|_{\text {ModelB }}=\frac{\phi 3}{\Gamma^{2}}[\cosh (\Gamma Y)-\cosh (\Gamma S)]+\frac{B i D a \psi}{\Gamma^{2}}\left[\frac{Y^{2}}{2}-\frac{S^{2}}{2}\right]- \\
\frac{B i C \psi}{Z^{2}\left(Z^{2}-\Gamma^{2}\right)}[\cosh (Z Y)-\cosh (Z S)],
\end{gathered}
$$

1 where

$$
\begin{gathered}
\phi 1=\frac{\Gamma}{\sinh (\Gamma S)}\left[\frac{k}{\gamma}-\frac{B i D a \psi}{\Gamma^{2}} S-\frac{C \psi\left(Z^{2}-B i\right)}{Z\left(Z^{2}-\Gamma^{2}\right)} \sinh (Z S)\right], \\
\phi 2=\frac{\Gamma}{\sinh (\Gamma S)}\left[\frac{1}{\gamma}-\frac{B i D a \psi}{\Gamma^{2}} S+\frac{B i C \psi}{Z\left(Z^{2}-\Gamma^{2}\right)} \sinh (Z S)\right], \\
\phi 3=\cosh (\Gamma S)\left[-\frac{\phi 1}{\Gamma^{2}}-\frac{\phi 2}{B i}\right]+\frac{B i D a \psi}{\Gamma^{2}}\left(-\frac{1}{B i}-\frac{S^{2}}{2}\right)+\frac{B i C \psi}{Z^{2}\left(Z^{2}-\Gamma^{2}\right)} \cosh (Z S), \\
\psi=\frac{k}{\bar{U}}\left(1+\frac{1}{\gamma}\right), \\
\Gamma=\sqrt{B i(1+k)} .
\end{gathered}
$$

\section{2.4. Validation}

3 To validate the present exact solutions, temperature distributions in the solid and fluid phases are

4 evaluated for a channel fully filled with a porous material. In Eqs. (33) and (34) for model A and Eqs.

5 (41) and (42) for model B, $S$ is set equal to one. The obtained results are compared against the exact 6 solutions derived by Marafie and Vafai [12] and Yang and Vafai [7]. The excellent agreement between 7 these solutions, shown in Fig. 2, confirms the validity of the presented analysis.
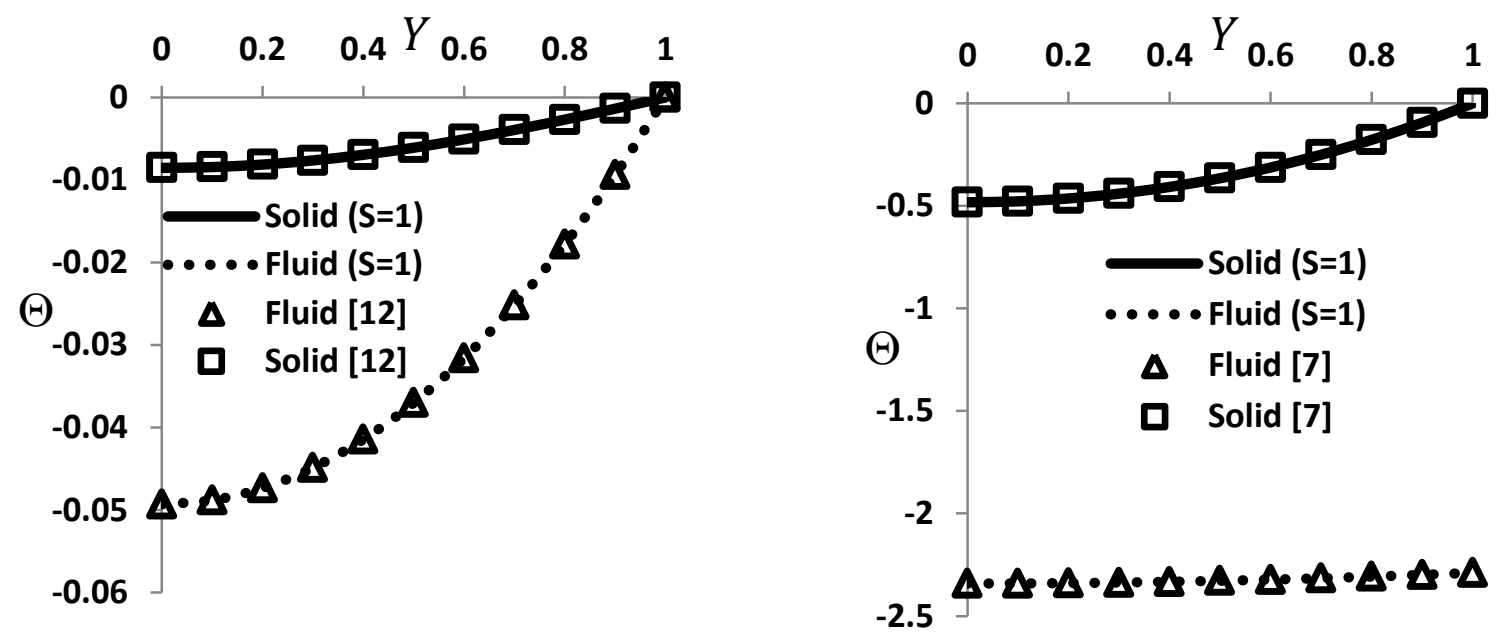

(a)

(b) 
Fig. 2. Temperature distributions in the fluid and solid phases for $S=1, k=0.01, B i=10$ and $D a=10^{-4}$. Solid lines: present solutions, symbols: exact solutions in [7, 12], (a) model A, Eqs. (33) and (34) and, (b) model B, Eqs. (41) and (42).

\section{3. Conclusions}

3 Temperature fields of the solid and fluid phases in a channel partially filled with a porous medium under

4 local thermal non-equilibrium condition were investigated analytically. The coupled partial differential

5 equations for the transport of momentum and energy in a fluid flow through a porous medium, along with

6 the energy balance of the solid matrix were considered. These were first converted to a set of decoupled

7 ordinary differential equations and were subsequently solved analytically. Further, two distinctive thermal

8 boundary conditions (the so-called models A and B) were applied to the interface between the porous

9 medium. Model A assumes that heat is divided between the two phases on the basis of their effective 10 conductivities and their corresponding temperature gradients. Model B, however, assumes that both 11 phases at the interface receive the same amount of heat flux. Exact solutions were developed for the solid

12 and fluid phases. These solutions are of direct use in the analysis of heat transfer enhancement as well as 13 the validation of numerical simulations under varying parameters. In addition, the present set of results 14 can be used as a supplement in the numerical simulations of the more involved problems. This is to 15 reduce the computational burden of solving the momentum and energy equations. An example of such 16 problems is the analysis of reactive flows in porous media.

17

18 Nomenclature

$\begin{array}{llll}a_{s f} & \text { Interfacial area per unit volume of porous } & x & \text { Longitudinal coordinate }(\mathrm{m}) \\ & \text { media }\left(\mathrm{m}^{-1}\right) & & \\ A, B & \text { Constant parameter defined by Eq. }(16 \mathrm{~b}, \mathrm{c}) & y & \text { Transverse coordinate }(\mathrm{m}) \\ B i & \text { Biot number, } & Y & \text { Dimensionless } y \text { coordinate, } y / \mathrm{h}_{0}\end{array}$




\begin{tabular}{|c|c|c|c|}
\hline$C$ & Constant parameter defined by Eq. (17b) & $Z$ & Constant parameter, $\sqrt{1 / D a}$ \\
\hline$C_{p}$ & Specific heat of the fluid, $\left(\mathrm{J} \mathrm{Kg}^{-1} k^{-1}\right)$ & $\begin{array}{l}\text { Greek } \\
\text { symbols }\end{array}$ & \\
\hline$D a$ & Darcy number, $K / \mathrm{h}_{\mathrm{o}}{ }^{2}$ & $\gamma$ & $\begin{array}{l}\text { Ratio of wall heat flux to the } \\
\text { heat flux at the interface, } \\
q_{w} / q_{\text {interface }}\end{array}$ \\
\hline$h_{s f}$ & $\begin{array}{l}\text { Fluid to solid heat transfer coefficient ( } \mathrm{W} \mathrm{m}^{-} \\
\left.{ }^{2} k^{-1}\right)\end{array}$ & $\Gamma$ & $\begin{array}{l}\text { Constant parameter defined by } \\
\text { Eq. (43d) }\end{array}$ \\
\hline $\mathrm{h}_{0}$ & Height of the channel (m) & $\varepsilon$ & Porosity of the porous medium \\
\hline$h_{p}$ & Porous substrate thickness (m) & $\Theta$ & Dimensionless temperature \\
\hline$K$ & Permeability of the porous medium $\left(\mathrm{m}^{2}\right)$ & $\mu$ & Viscosity $\left(\mathrm{Kg} \mathrm{m}^{-1} s^{1}\right)$ \\
\hline$k$ & $\begin{array}{l}\text { The ratio of solid effective thermal } \\
\text { conductivity to that of the fluid, }(1-\varepsilon) k_{s} /\left(\varepsilon k_{f}\right)\end{array}$ & $\rho$ & Density, $\left(\mathrm{kg} / \mathrm{m}^{3}\right)$ \\
\hline$k_{f}$ & Thermal conductivity of the fluid $\left(\mathrm{W} \mathrm{m}^{-1} k^{-1}\right)$ & $\xi$ & $\begin{array}{l}\text { Constant parameter used in Eq. } \\
\text { (34) }\end{array}$ \\
\hline$k_{f, e f f}$ & Effective thermal conductivity of the fluid, $\varepsilon k_{f}$ & $\psi$ & $\begin{array}{l}\text { Constant parameter defined by } \\
\text { Eq. (43d) }\end{array}$ \\
\hline$k_{s}$ & Thermal conductivity of the solid $\left(\mathrm{W} \mathrm{m}^{-1} k^{-1}\right)$ & $\phi 1, \phi 2, \phi 3$ & $\begin{array}{l}\text { Constant parameter defined by } \\
\text { Eq. }(43 a, b, c)\end{array}$ \\
\hline$k_{s, e f f}$ & $\begin{array}{l}\text { Effective thermal conductivity of the solid, (1- } \\
\varepsilon) k_{s}\end{array}$ & Subscripts & \\
\hline $\mathrm{O}_{1}, \mathrm{O}_{2}$ & Constant parameter defined by Eq. $(40 b, c)$ & eff & Effective property \\
\hline$p$ & Pressure $(\mathrm{Pa})$ & $f$ & Fluid \\
\hline$q$ & Heat flux $\left(\mathrm{W} \mathrm{m}^{-2}\right)$ & $f 1$ & Fluid in the clear region \\
\hline$S$ & Ratio of the porous medium thickness to the & $f 2$ & Fluid in the porous medium \\
\hline
\end{tabular}




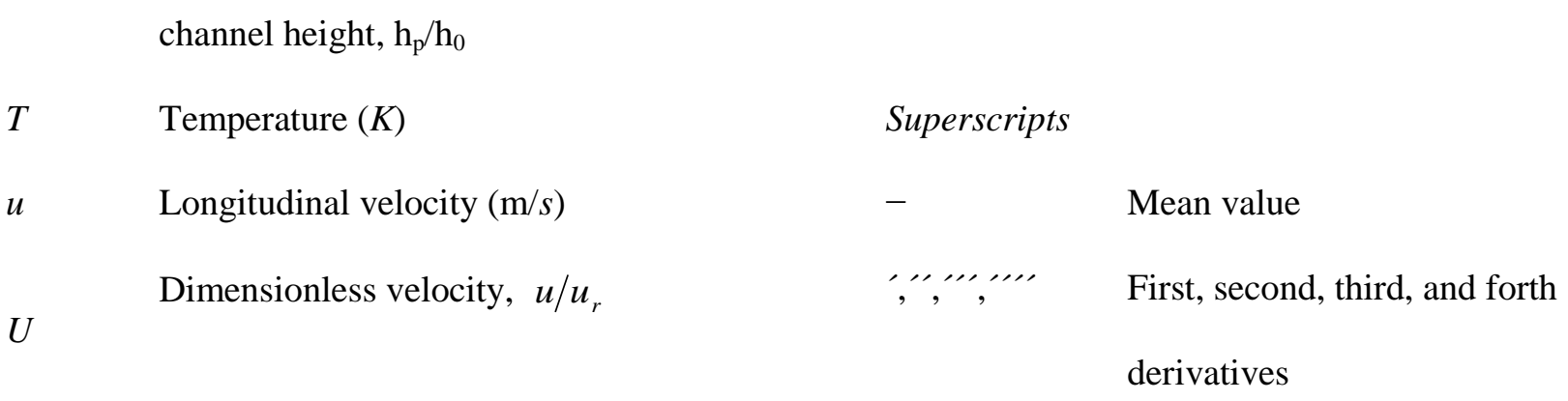

\section{References}

[1] Webb, R.L., Kim, N. Principles Of Enhanced Heat Transfer, 2nd edition, New York, Taylor \& Francis, 2005.

[2] Vafai, K., Handbook of porous media, 2nd edition, New York, Marcel Dekker, 2000.

[3] Mahmoudi, Y., Maerefat M. Analytical investigation of heat transfer enhancement in a channel partially filled with a porous material under local thermal non-equilibrium condition. Int. J. Thermal Sciences 2011; 50: 2386-2401.

[4] Maerefat, M., Mahmoudi, S.Y., Mazaheri, K., Numerical simulation of forced convection enhancement in a pipe by porous inserts. Int. J. Heat Transfer Engineering 2011; 32: 45-56.

[5] Yang, K., Vafai, K. Restrictions on the validity of the thermal conditions at the porous-fluid interface: an exact solution. ASME J. Heat Transfer 2011c; 133: 112601-12.

[6] Yang, K., Vafai, K. Analysis of heat flux bifurcation inside porous media incorporating inertial and dispersion effects - an exact solution. Int. J. Heat Mass Transfer 2011b; 54: 5286-5297.

[7] Yang, K., Vafai, K. Analysis of temperature gradient bifurcation in porous media - an exact solution. Int. J. Heat Mass Transfer 2010; 53: 4316-4325.

[8] Alazmi, B., Vafai, K. Constant wall heat flux boundary conditions in porous media under local thermal non-equilibrium conditions. Int. J. Heat Mass Transfer 2002; 45: 3071-3087.

[9] Amiri, A., Vafai, K., Kuzay, T.M. Effect of Boundary Conditions on Non-Darcian Heat Transfer Through Porous Media and Experimental Comparisons. Numerical Heat Transfer Journal 1995, 
Part A; 27: 651-664.

[10] Alazmi, B., Vafai K. Analysis of fluid flow and heat transfer interfacial conditions between a porous medium and a fluid layer. Int. J. Heat and Mass Transfer 2001; 44: 1735-1749.

[11] Lee, D.Y., Vafai K. Analytical characterization and conceptual assessment of solid and fluid temperature differentials in porous media. Int. J. Heat Mass Transfer 1999; 42: 423-435.

[12] Marafie, A., Vafai, K. Analysis of non-Darcian effects on temperature differentials in porous media. Int. J. Heat Mass Transfer 2001; 44: 4401-4411. 